\title{
BUILDING UNDERSTANDING IN ASYCHRONOUS DISCUSSIONS: EXAMINING TYPES OF ONLINE DISCOURSE
}

\author{
Seungyeon Han, Ph.D. \\ Hanyang Cyber University, Seoul, Korea
}

Janette R. Hill, Ph.D.

Instructional Technology, University of Georgia

\begin{abstract}
This article describes a study exploring how students' learning is reflected in asynchronous online discussion. The study examined how online discourse contributes to the learning process. In examining online group discussions using discourse analysis, five different types of discourse were identified: goal setting, reflection, connection, original reformulation, and re-direction. With the different types of discourse, we explained how each message or a threaded message facilitated the discussion, particularly in terms of collaborative efforts to achieve the goal(s). The nature of each type of discourse was illustrated, including how different strategies were identified in the data and how different results of discussion were demonstrated in the data. We also describe the methodological issues related to the analysis of online discourse and discuss implications for research and practice.
\end{abstract}

\section{KEYWORDS}

Asynchronous Discussion, Learning Process, Learning Outcomes, Discourse Analysis, Collaboration

\section{INTRODUCTION}

The Web continues to offer new opportunities for educational practice. Researchers studying the use of the Web for learning are also beginning to explore the larger implications of this technology. Researchers have indicated that the socio-cultural implications of the use of the Internet are important to explore. Recent studies have reported the benefits of using the Internet for collaborative learning [1, 2, 3, 4]. Two areas of particular interest are social interaction and co-construction of knowledge [5,6]. Researchers in these areas indicate that Web-based learning environments (WBLE) enhance social interaction amongst participants and provide opportunities for mutual construction of knowledge [6, 7].

Despite the growing interest in and research related to collaborative learning in online environments, the nature of the social interaction and the processes associated with the mutual construction of knowledge remains largely undiscovered. For example, a variety of interaction types are well elaborated in the literature [8,9], but most descriptions of the interactions exist in describing relationships (e.g., learnerlearner, learner-instructor, learner-content, instructor-content, learner-interface) in various technologymediated contexts. These descriptions enable us to better understand where interaction exists and what interaction occurs. However, there is little exploration in terms of the qualitative aspects of interaction such as how the interaction occurs, how the interaction is situated in a variety of contexts, and the distinctive nature of each type of interaction (e.g., how learner-learner interaction is reflected in online discussion boards). 
One way research related to this area might be accomplished is to explore the interactions that occur during online learning. The discussion generated and transcripts created by online learning might yield important insights related to how knowledge creation and distributed learning occurs online. As analysis of online discourse is an emerging field [10], we have a limited understanding regarding the types of messages generated in the course of a discussion. We need to further explore how the nature of online discourse reflects collaborative learning whereby technology, group efforts, tasks, and time interact dynamically within the learning context.

The purpose of this study was to investigate the nature of group discourse in a Web-based learning environment supported by computer conferencing. The primary research question guiding the study was "how does online discourse contribute to the learning process?" We begin with a review of the foundations of social learning theory, followed by a description of the study and major findings. We end with a discussion of implications for research and practice.

\section{CONCEPTUAL FRAMEWORK}

How knowledge is acquired and shared is an important aspect of any learning situation. It is particularly important in online learning where the very nature of what is learned and how it occurs has been called into question by researchers (e.g., [11, 12]). Social learning theory offers a unique insight from which to view online learning. From this perspective, learning is situated in a specific social context [13, 14], and cognition is distributed across individuals, tools, and artifacts [15, 16, 17]. In an online learning context, knowledge is socially constructed in primarily written formats and learning is enabled via various means of communication. Knowledge and learning occur in a gradual convergence through interactive communication and facilitated collaboration.

From a situated learning perspective, there is no separation of knowing from that which is known; rather, there is an assumption that practice, meaning, and identity constitute and are constructed within a context, suggesting an interweaving among practice, meaning, and context [18, 19]. The online discourse generated by asynchronous discussion cannot be separated from the technology (i.e., online course management systems) or the social context (i.e., class). The discourse is partially controlled by technological affordances, the learner(s), and the context. The interactions are dynamic, such that the writing of one person can only be described and understood in relation to the response of the other persons, and in relation to the situational and temporal circumstances in a community of learners [15, 20].

The distinction between technological and social dimensions provides a unique view from which to examine the complex and multifaceted nature of the learning process in a WBLE. There are both technological and social dimensions of learning $[16,17]$. The technological dimension emerges from a specific context enabled by the technological affordances. The social dimension is constructed from the collaboration between the participants.

\section{A. Technological Dimension in Asynchronous Discussion}

One of the greatest potentials for technology-mediated communication is its ability to provide unique affordances, in this instance, an infrastructure to enable group and collaborative learning [21, 22, 23]. According to Pea [16], "affordance refers to the perceived and actual properties of a thing, primarily those functional properties that determine just how the thing could possibly be used” (p. 51). Thus for example, in asynchronous discussion boards, the affordances of computer and Internet technologies enable communication via the generation of discussion messages amongst participants. 
Computer conferencing typically involves interaction amongst a group of participants. A user can log on and read the contributions (i.e., messages) of other members of the group, respond to the message(s) posted or create a message for a new thread. Most current conferencing tools (e.g., Blackboard $®$ ) allow the inclusion of other media like pictures and links to web pages or other information in a message. The systems also include additional features like the ability to organize the messages by author, topic theme, keywords, or chronological order.

A threaded discussion is a simple form of hierarchically structured written-text provided by computer conferencing systems [24]. A threaded discussion usually shows the list of all the messages with subject headings, enabling a structuring of messages by topic. A common use of the threaded discussions in learning contexts involves a participant (i.e., instructor, student) specifying a topic for discussion in advance and others posting their response messages containing opinions, comments, or questions about the topic. The individual messages are thus organized by topics [25] that emerge in the discussion.

One aspect of online learning that seems quite different from face-to-face learning involves class discussion. Online class discussion does not necessarily evolve sequentially through time, as classroom discussion does, but rather grows over time from multiple conceptual perspectives in many dimensions all at once [26, 27]. The nature of the interactions assists in enabling the evolution of the discussion; however, the affordance of the technology also plays a role. Responses to messages may be delayed because of the asynchronous nature of the conversation. Time between the postings of messages among participants may range from several seconds to several days or longer depending on the length of time that the discussion forum or thread is available to its participants.

\section{B. Social Dimension in Asynchronous Discussion}

From a social learning perspective, knowledge is socially constructed, and language is constructed and situated within a social context [28, 29]. "A primary function of human language is to scaffold the performance of social activities and to scaffold human affiliation within culture and social groups and institutions” [29, p. 1]. Therefore, writing is used to construct and reconstruct knowledge by explaining our beliefs in community [30]. Knowledge building and/or meaning making in learning is an integral part of a social context and culture when we explore the relationship among contents of text and the context where the meaning is created [28,31]. If we are to support learning via discourse in an online learning context, we need to establish the construction of knowledge as a social activity, with new ideas and information brought into the discourse of a community that shares goals for knowledge advancement and recognizes the contributions of multiple participants.

In order to examine a complex construct such as interaction, researchers benefit from carefully examining the context within which the interaction is taking place. An interesting aspect of asynchronous online learning is that interaction is mainly constructed in written form. It has been suggested that asynchronous online discourse is a new kind of language showing hybrid features of both spoken and written language [27, 32, 33]. Language in online discourse is typed and therefore, like writing; however, it contains exchanges similar to that found in face-to-face conversation, which are "often rapid and informal," and therefore like talk. Thus, "it reads like and to a certain extent acts like conversation" [27, p. 2].

The nature of discourse is complex in any circumstance. In online contexts, the complexity is even more pronounced. It is important to consider many dimensions in the exploration of online discourse for learning. Two discussed here included the technological and social. It is also important to examine how knowledge is constructed, as well as the theories associated with learning, as we seek to extend our understanding. These principles were used to guide the study described in the next section. 


\section{METHODOLOGY}

\section{A. The Context}

A qualitative case study was conducted in a Master's level course in a College of Education supported by a Web-based learning system (i.e., WebCT®, http://webct.com). In this study, three characteristics were used to select the case [36, 37]. First, the class was group-oriented; that is, the primary focus revolved around group activities and projects. Second, the primary delivery of the course was through asynchronous computer conferencing. Third, participants entered the course with both similarities (i.e., most participants are school teachers in $\mathrm{K}-12$ context) and differences (i.e., diverse web-based learning experience).

The implementation of the course took place in a research university in the South during a short session in the summer (four weeks). The participants consisted of the university instructor $(n=1)$, doctoral students as facilitators $(n=2)$, and $K-12$ teachers and school library media specialists as students $(n=23)$. At the time of the study, most students $(n=21)$ had experience (i.e., one or more courses) learning in an online environment. Adhering to national trends in school library media programs, the participants were predominantly female $(\mathrm{n}=22)$ and Caucasian $(\mathrm{n}=21)$.

There were six different project groups $(n=3-4)$, with two project groups paired as a discussion group. Consequently, there were three different discussion groups ( $\mathrm{n}=6-8$ participants) using the discussion boards throughout the implementation of the course. Each group was assigned in a specific discussion forum for their group with two discussion topics per week. A single discussion topic contained multiple discussion threads.

The first author of this paper was one of the facilitators and supported student participants to complete individual and group projects and assisted with the discussion. She also had primary responsibilities for data collection and analysis. The second author of this paper was the major instructor and was primarily responsible for course design, implementation, and facilitation.

The Instructional Design course provided an introduction to the instructional design process within a hands-on setting. The course offered sixteen class meetings within a blended technology enhanced learning environment. Students were expected to complete individual activities (i.e., ID reflection, reflection on course material readings), with a culminating group project (i.e., IDAs, Instructional Design Activities).

During the course, participants experienced a variety of instructional methods, such as face-to-face workshops, synchronous online chat, and asynchronous discussion. Face-to-face workshops provided logistical support, a preview of course content, and opportunities to work on group tasks within a classroom and computer lab. Synchronous online chat sessions (i.e., virtual office hours) offered opportunities to ask questions and discuss the course content.

Asynchronous discussions forums allowed participants to discuss multiple topics (i.e., learner analysis, instructional strategies, evaluation) throughout the course between face-to-face workshops. The asynchronous discussion board enabled a variety of interactions to assist students' learning in several ways (e.g., sharing information, presenting and providing feedback on each others' work, discussion course related topics, and reflection on the instructional design process). 


\section{B. Data Generation and Analysis}

The primary data used for this study were the transcripts from the discourse captured from the asynchronous discussion board. There were 150 discussion topics, with a total of 621 messages generated across all participants during the implementation of the course. The content of the messages ranged from course topics to technological support (e.g., how do I make the link in a Web page?), to more socially focused messages (i.e., sharing stress tips). The transcripts of the discussions board were collected as a compiled text file at the end of the course for analysis.

The online learning tool controlled the structure of the threaded discussion. A generic feature of most discussion tools is that the discussion is displayed as a hierarchical and linear process. Figure 1 represents an example of a threaded discussion in the WebCT ${ }^{\circledR}$ system. By providing threading capabilities, conference discussions remain relatively structured and coherent, and users can easily track the evolution of group discussions around specific topics.

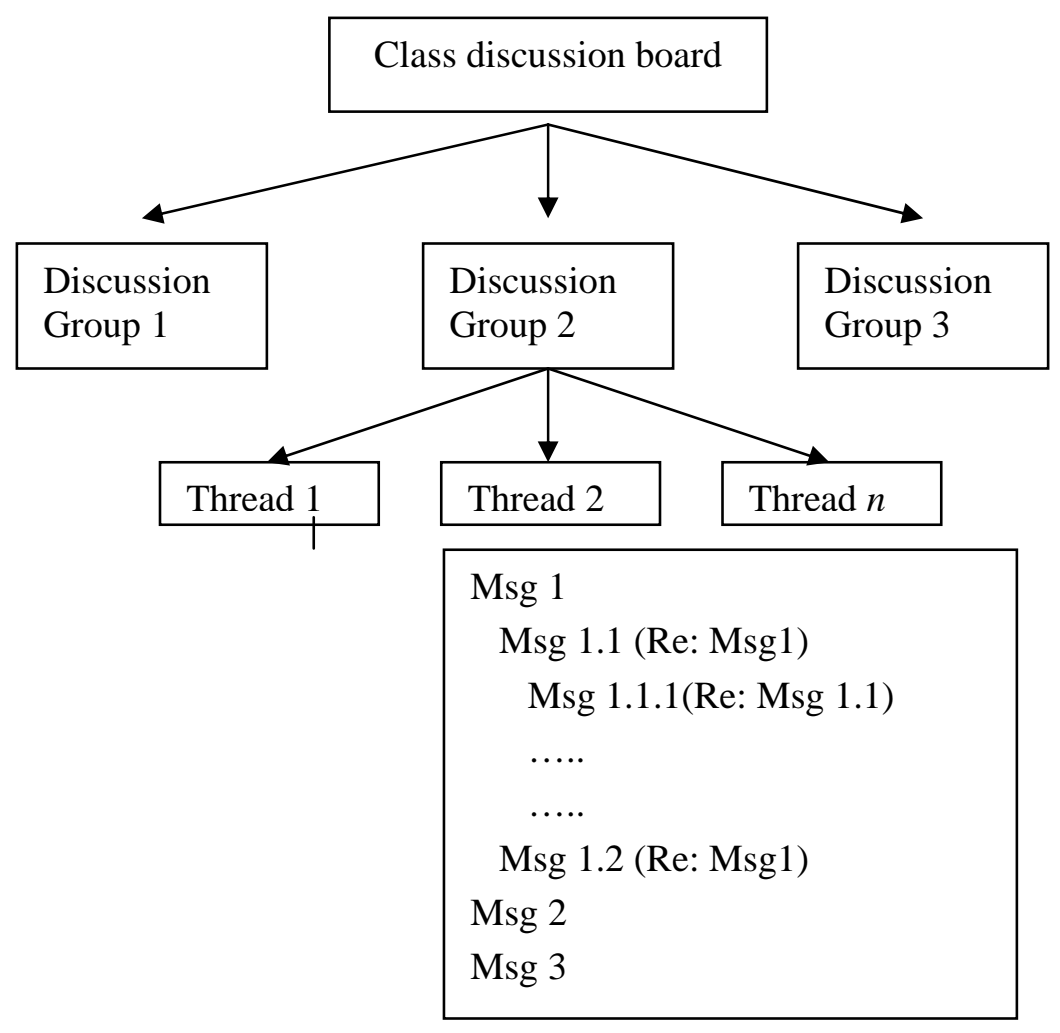

Figure1. The Structure of Asynchronous Discussion Groups

One discussion group $(\mathrm{n}=8)$ was selected for in depth analysis of the nature of the learning process. This discussion group, comprised of two different project groups, was selected based in several factors. First, the personal background and current position of each participant varied, enabling multiple viewpoints and cultures to manifest during the discussion. Second, the group members had different experiences in online learning environments. The majority of the group members $(n=6)$ had three or more either purely online learning or a mixture of face-to-face and online learning (i.e., hybrid) courses. One participant had two online/hybrid courses and one participant had no experience with online or hybrid courses. Third, this discussion group had a rich set of discussion in terms of number of messages posted in the forum. During the implementation of the course (i.e., four weeks), the group generated 170 messages directly related to 
the course content, an average of 21 per participant. The total number of messages was $16 \%$ more than the next closest group who generated 105 messages. Table 1 presents an overview of the participants.

\begin{tabular}{|c|c|c|c|}
\hline Name & Gender & Experience & Current Job \\
\hline Alice & Female & $3^{\text {rd }}$ online/hybrid class & Media specialist in elementary school \\
\hline Annette & Female & $\begin{array}{l}4^{\text {th }} \text { online/hybrid class } \\
\text { (The last class of the program) }\end{array}$ & $\begin{array}{l}\text { Coordinator of information services at a } \\
\text { K-12 private school }\end{array}$ \\
\hline Debb & Female & $3^{\text {rd }}$ online/hybrid class & Elementary school teacher \\
\hline Jamie & Female & $3^{\text {rd }}$ online/hybrid class & Media specialist in elementary school \\
\hline Jane & Female & $3^{\text {rd }}$ online/hybrid class & $\begin{array}{l}\text { Recruitment specialist in the county school } \\
\text { district }\end{array}$ \\
\hline Julie & Female & $4^{\text {th }}$ online/hybrid class & High school teacher \\
\hline Karin & Female & $1^{\text {st }}$ class in the program & Media specialist in elementary school \\
\hline Sean & Female & $2^{\text {nd }}$ online/hybrid class & Elementary school teacher \\
\hline
\end{tabular}

Table 1. Overview of Participants

\section{Data Analysis Procedure}

Data analysis included the 170 messages generated by the discussion group, consisting of five discussion topics (i.e., needs assessment and learner analysis; objectives and assessment; strategies, activities and materials; implementation and evaluation; and instructional consultation) with 17 threads in the discussion topics. Detailed analysis was implemented in individual messages; the fundamental unit of analysis was a thread of the discussion. Individual messages were analyzed within the context of the thread, and the threads were cross-examined during the analysis.

Preliminary analysis of the data started when the data was generated. In depth analysis was a complex and multi-layered process and took place over several months. Figure 2 presents an overview of the procedure. 


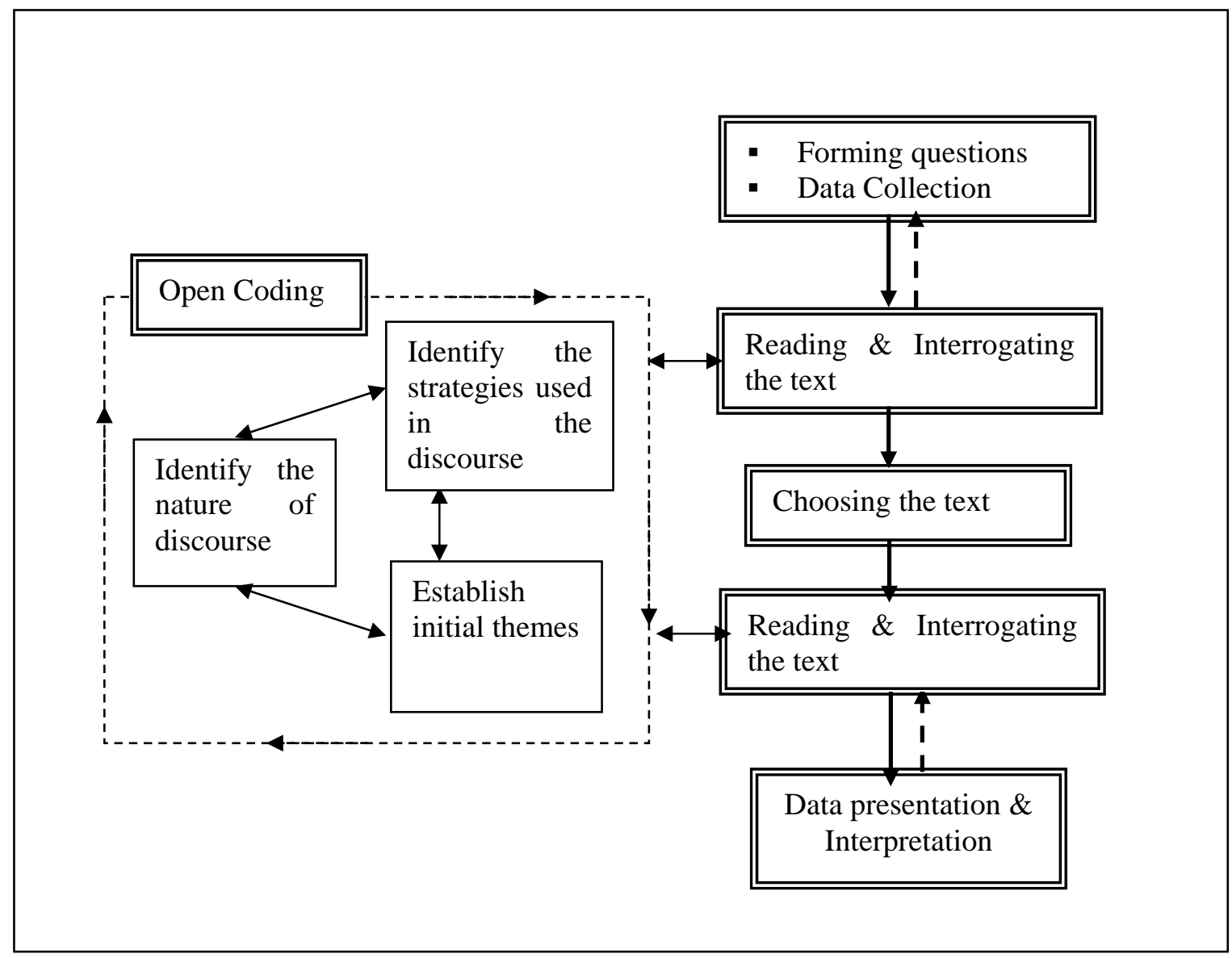

Figure 2. Data Analysis Procedure

Discourse analysis was the primary strategy used in analyzing the transcripts of the asynchronous discussion. Discourse in this study is defined as “a means of constituting cognition” [38, p. 2]. A fundamental assumption of discourse analysis is that language is a social consequence, and language scaffolds the social interaction within culture of community [29, 30]. Discourse is situated in specific context, adapted to the technological and social affordances of the context [16, 17, 38]. Knowledge construction and/or meaning making are not separate from the social context and culture in which the construction occurs. A primary purpose of discourse analysis is to explore the relationship among the content of the texts and context where in the meaning is situated [28, 29, 31].

Researchers have used diverse analytic methods in the research of electronic discourse generated by computer conferencing. For example, from the early exploration of interaction and collaboration in asynchronous discussion, content analysis was one of the methods used (e.g., Gunawardena, Lowe, \& Anderson, 1997; Henri, 1992) (see Bauer, 2001 for a review of content analysis). Content analysis of the transcript of discussion includes establishing theory based analysis protocols, coding the data, illustrating how each category applied to the data, and reporting the quantified results (i.e., how many message were generated in each category).

Discourse analysis used in this study is different from other methods that have been used in this area (e.g., content analysis) for several reasons. First, the focus of analysis was not just the content presented in an individual message. The focus was the meaning situated in a context (i.e., threads, topics) and connections between the individual messages. Moreover, we examined the threads of the discussion in regard to how 
message(s) contributed to continuing the discussion. Second, this study excludes the quantification of the results. That is, enumerating the numbers for each category was not essential. Rather, we presented how individual message can be categorized. As other researchers suggested, in the early use of established analysis protocols, counting numbers would be useful to validate the code itself [39]. Yet, we believe as [12] do that the transcript data is complex in nature; calculation may not be as supportive for understanding the essence of the data. Third, the types identified in this study (see Table 2) are grounded in the data. Meaning interpretation was guided by and substantiated with the literature, as well as illustrated from the data.

There are multiple views and foundations of discourse analysis (see review of discourse analysis as methodology [40, 41]). As described above, this study was guided by a situated learning perspective (e.g., [38]). The authors found Gill [31] and Wood and Kroger [41] were useful to begin research in this area in terms of analytic strategies, which includes: forming a question, reading and interrogating the data for establish initial themes, choosing the text for more in depth analysis, reading and interrogating in detail, and data presentation and interpretation.

The first step in the process involved reading and interrogating (i.e., cross examining the threads) all transcripts of the asynchronous discussion. Several steps were involved in the open coding process. By analyzing individual messages, the first rounds of analysis were directed at establishing initial themes. The nature of each message was coded (e.g., define goals, present individual reflection) and the strategies used in writing the messages were identified (e.g., questioning, argument).

Next, we examined the connections between messages; that is, how one message was read and responded to by other participants. This enabled us to gain an initial understanding of how the learning process was presented in individual messages (e.g., idea initiation, meaning negotiation) and the result of the discussion (e.g., shared goals, shared accounts). Finally, several main categories from the data were eliminated or collapsed together as they were considered overlapping or repetitive.

We used three different strategies to check reliability: deviant case analysis, coherence, and peer examination [31]. First, a detailed analysis of transcripts was performed to identify the 'go against' messages (what [31] refers to as "deviant cases analysis"). Second, to check the coherence of the analysis, earlier studies similar to this study were reviewed to help confirm the consistency of the analysis techniques used (e.g., [10, 42]). Finally, peer examination was conducted to confirm the analysis methods and conclusions.

\section{FINDINGS}

The focus of the discourse analysis in this study was the learners' collaborative efforts. Specifically, we examined how different discourse types facilitated the discussion, and also helped enable collaborative learning. Five types of discourse were identified in this study: goal setting, reflection, connection, original reformulation and re-direction. Table 2 provides a summary of the types of discourse, including the nature of communication, process of learning, discourse strategies, and the results of the discussion. 


\begin{tabular}{|c|c|c|c|c|}
\hline $\begin{array}{l}\text { Type of } \\
\text { discourse }\end{array}$ & $\begin{array}{l}\text { Nature of } \\
\text { communication }\end{array}$ & Process of learning & Discourse Strategies & $\begin{array}{l}\text { Results of } \\
\text { discussion }\end{array}$ \\
\hline Goal setting & - $\quad$ Establish goal(s) & $\begin{array}{l}\text { - } \quad \text { Idea initiation } \\
\text { Goal } \\
\text { concretizing }\end{array}$ & $\begin{array}{ll}\text { - } & \text { Questioning } \\
\text { - } & \text { Summary } \\
\text { - } & \text { Monitoring } \\
\text { - } & \text { Quote/comments }\end{array}$ & - Shared goals \\
\hline Reflection & $\begin{array}{ll}\text { - } & \text { Represent } \\
\text { individual } \\
\text { understanding }\end{array}$ & - Reflection & $\begin{array}{ll}\text { - } & \text { Illustration } \\
\text { - } & \text { Explanation } \\
\text { Citation }\end{array}$ & $\begin{array}{l}\text { - } \quad \text { Shared } \\
\text { account }\end{array}$ \\
\hline Connection & $\begin{array}{l}\text { Associate } \\
\text { individual } \\
\text { understanding } \\
\text { within a } \\
\text { collaborative } \\
\text { context }\end{array}$ & $\begin{array}{ll}\text { - } & \text { Exploration } \\
\text { Sharing } \\
\text { perspectives }\end{array}$ & $\begin{array}{l}\text { - } \text { Quote/comments } \\
\text { - } \text { Agestioning } \\
\text { Agreement }\end{array}$ & $\begin{array}{ll}\text { - } & \text { Mutual } \\
\text { agreement }\end{array}$ \\
\hline $\begin{array}{l}\text { Original } \\
\text { Reformulation }\end{array}$ & $\begin{array}{l}\text { Challenge } \\
\text { existing } \\
\text { knowledge and } \\
\text { create knew } \\
\text { knowledge }\end{array}$ & $\begin{array}{ll}\text { - } & \text { Integration } \\
\text { - Extension } & \text { Expand }\end{array}$ & $\begin{array}{ll}\text { - } & \text { Questioning } \\
\text { - } & \text { Reviewiening }\end{array}$ & $\begin{array}{l}\text { - } \quad \text { Collective } \\
\text { knowledge }\end{array}$ \\
\hline Re-direction & $\begin{array}{l}\text { - Alter/modify } \\
\text { goal(s) }\end{array}$ & - Conversion & $\begin{array}{ll}\text { - } & \text { Quote/comments } \\
\text { - } & \text { Summary } \\
\text { Questioning }\end{array}$ & 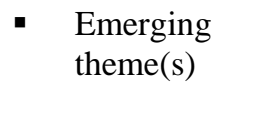 \\
\hline
\end{tabular}

Table 2. Types of Discourse

\section{A. Goal Setting}

The first type of discourse demonstrated in the data was "goal setting." The nature of the communication during goal setting was to establish the goal(s) of discussion, thus framing the topic(s) of the discussion. In this context, the students were engaged in idea initiation using guiding questions from the instructor or other students during goal setting. Participation in the asynchronous discussion was a requirement of this class; consequently, one of the common goals in this context was engagement of discussion (i.e., reading and posting messages). Figure 3 displays one example of a "goal setting" message, in this instance one generated by the instructor.

1 Message no. 181

2 Posted by Jo on Monday, May 19, 2003 8:14am

3 Subject Classifying (?) a Learning Task

4 Greetings, all! 
5 Here is something to help launch our discussion for the next day or so...

6 Based on some of the discussion and questions that have been posed, it seems that 7 the idea of "classifying” a learning task may seem a bit "odd." (note: you may not 8 think this is true - just basing it on some comments that have been posed!). Yet, 9 there are several taxonomies that are specifically designed just for this activity (e.g., 10 Bloom, Gagne). Why is it important to classify learning tasks? And how might 11 classification help you with the work you are doing now re: your objectives and 12 assessment?

13 best- Jo

Message no. 212[Branch from no. 181]

Posted by Julie on Monday, May 19, 2003 8:04pm

Subject Re: Classifying(?) a Learning Task

I guess it does seem a bit unnatural to officially classify a learning task, but I think that most teachers do it naturally. I think that the purpose is to make sure that your lessons are leading to higher order thinking and you aren't simply teaching the same general skills over and over again with different content. It is also important so you teach in a logical order...that a higher order skill is not taught before a lower one (does that make sense?).

Julie

Message no. 215[Branch from no. 212]

Posted by Alice on Monday, May 19, 2003 8:12pm

Subject Re: Classifying(?) a Learning Task

I think of classifying a learning task when the teachers know the curriculum and do their lesson plans. They know what they will be doing: the tasks that have worked before, incorporate new ideas and adjusting to the group of kids they are directing it to. I agree with Julie that the teachers do this without the writing of the task down to the detail we are doing. I believe their bases would be the QCCs or AKS ... knowing they have to teach certain curriculum units and if they preplan with others they have that much more experience to draw from.

Alice

[the rest of discussion thread omitted]

Figure 3. An Example of a Goal Setting Message

The goal setting displayed in the text of Figure 3 includes the planning and defining of the goal, what Bereiter and Scardamalia [43] refer to as "goal concretizing." The instructor (Jo) planned the discussion before the discussion began and provided a "guiding question" (e.g., "what are the benefits of inductive and deductive assessment?") during the discussion to get the dialogue started (e.g., "why is it important to classify learning tasks?"). In the larger class structure, the global goal was framed by the instructor (i.e., to discuss topics related to objectives and assessment in instructional design process), and then she concretized the goal (i.e., elaborate the classification of learners' tasks) to initiate and facilitate the discussion. 
During the goal concretizing process, the instructor used different strategies: summary, questioning, and monitoring. For example, she provided her view on what students have discussed in previous topics in the beginning of the messages (lines 6-8) and framed the questions for present discussion (lines 10-12). To present her perspective on this topic, she introduced the idea with related resources (line 11). The result of this type of discourse was "shared goals" as demonstrated in the discussion responding to this question.

In the example described above, the goal was shared and provided a common ground for the discussion. As the participants continued the discussion, the topic was elaborated with individual learner's perspectives and extended to other topics. Figure 3 illustrates how the participants created and posted message based on shared goals (i.e., engagement of discussion and respond to the guiding question). Shared goals can be observed in a group of messages as the discussion evolves.

\section{B. Reflection}

A reflection message can be described as a reflection of an individual's experience and knowledge. The nature of communication is to present an individual's understanding of a given topic. A reflection message was often found in the beginning of the discussion thread. Participants either responded to the guiding question posted in the discussion board by facilitators or other students, or quoted the guiding question on the course website then created their individual understanding on the questions and topics. The following excerpt is an example of reflection (see Figure 4).

1 Message no. 284 (4860-4872)

2 Posted by Jamie on Tuesday, May 20, 2003 8:18pm

3 Subject Writing objectives

4 The example Turner gives for moderate level consultation (p. 132-133) really made

5 me think about how much gain there is in writing instructional objectives to too

6 detailed a level. I suspect that many teachers (especially the more experienced ones)

7 do the level of detail described, they just don't specify it that way.

8 Requiring it may put unnecessary stress on already overworked teachers.

9 Jamie

10 Message no. 76[Branch from no. 60]

11 Posted by Julie on Wednesday, May 14, 2003 11:16pm

12 Subject Re: Learner Analysis

13 I teach high school Language Arts in a very large school. This year my smallest 14 class is 27 and my largest is 32 . Unfortunately, I find that often the only way to 15 survive the workload and the paper load is to try to teach to the "average". I try to 16 design activities and assessments that target the greatest number of students, but I 17 know that I don't do a good enough job of catering to those students outside of the 18 average, but I do try. I often give several different options for projects, and try to 19 vary the types of assessment throughout the year, but some of what I assign is 20 dictated from higher up (i.e., certain number of practice essays per semester, etc.). 21 I think that if the curriculum was more concerned with depth of knowledge rather 22 than breadth, it would make it easier to tailor instruction to fit the individual 23 learner. 
24 Julie

Figure 4. An Example of a Reflection Message

The first example shows how a student reflected on her understanding of the given topic (i.e., different levels of writing objectives) and how she has found an idea about the topic (i.e., practical challenges in teachers practice). In composing message 284, Jamie translated the textbook into an authentic situation and applied reality to the description of theory. In the example, Jamie has used different strategies including citation (line 4), explanation (lines 4-6), and use of authentic examples (lines 7-8).

As shown in next example (message 76), Julie reports her personal experience as media specialist. She provided detailed information on the context (lines 13-14), stated the problem (lines 14-17), described the solution (lines 18-20), and then concluded her statement with a recommendation (lines 21-22). In a given topic (i.e., learner analysis), she explained what challenges she faced and resolved the identified challenge. As the above examples indicate, individual reflective thinking occurred while using the discussion board. The reflection indicated in the messages may lead to enhanced knowledge during the composing the message [44].

Julie clearly demonstrated a focus on self-reflection and presentation. When creating a reflection message, it appears that the participant's reflective thinking process is more focused on the individual's situation and not others. As Bereiter and Scardamalia [43] stated, from the notion of writing as a form of problem solving, "there is a great deal of soliloquy of the 'where am I variety', virtually no colloquy of the 'where are you' variety” (p. 301). However, when individual participants post their reflections in the discussion board, it is typically understood that the message will be read and responded to by others. Once it is shared among participants and other students present their reflective thinking on the message, the result of discussion becomes a shared account.

\section{Connection}

A connection message associates individual understanding within a collaborative context. This type of message attempted to converge different perspectives. Figure 5 shows an example of a thread of a discussion indicating how this type of discourse facilitates the discussion.

1 Message no. 346

2 Posted by Jane on Thursday, May 22, 2003 8:12am

3 Subject Goals vs Objectives

4 Do you guys think that sometimes we blur the line between goals and objectives?

5 For instance, I conducted a workshop for my women's organization called In Pursuit

6 of Goals. In that workshop we looked at overall goals as a bumper sticker or where

7 do you want to be, what do you want to achieve. Objectives were the steps to get

8 you to where you want to be. But my questions is how do you make sure you have

9 a distinction between goals and objectives or do you guys see them as the same thing?

10 Message no. 350[Branch from no. 346]

11 Posted by Karin on Thursday, May 22, 2003 9:51am

12 Subject Re: Goals vs Objectives

13 Certainly, I think it's a constant struggle to clarify your goals and objectives. Any 
14 group I have been involved with has had that same issue. Your have to continually 15 check your thinking and make sure you don't stray.

$16 \mathrm{~K}$ [initial]

17 Message no. 372[Branch from no. 346]

18 Posted by Debb on Thursday, May 22, 2003 7:20pm

19 Subject Re: Goals vs Objectives

20 Jane, the distinction between goals and objectives is sometimes (often) blurred for

21 me. I think your idea is good, that goals are the big ideas. You have to keep that

22 idea in the forefront of your mind. Debb

Figure 5. An Example of a Connection Message

In the first example, Jane (message 346) stated her current understanding of concepts (i.e., distinction between goal and objective, lines 4-8) using an authentic example and asked for other's perspectives. In general, this type of message appears to have enabled the participants to move from simple agreement to confirmation of the idea, creating an opportunity to reach mutual agreement. The interaction between the messages is instant and succinct. As depicted in Figure 5, the responses review or summarize the major point of the original message. Except for using direct/indirect quote strategies in the message, the length of a connection message was shorter in length than other types of messages. The thread of discussion tends to close in a short period, once the confirmation or mutual agreement on the idea is achieved.

In the next message (350), Karin made a confirmation (line 13) and reflected Jane's points in her experience (line 13-14). Debb also expressed a similar viewpoint to Jane (line 20-21) and provided her distinction between goals and objectives. Mutual agreement based on confirmation from others is summarized as "unclarity between goals and objectives have to constantly be checked" in this discussion thread. With a connection message, each participant has the responsibility to demonstrate the meaning and to account for that meaning through continuing discussion.

\section{Original Reformulation}

The nature of an original reformulation message can be characterized as a type of discourse that challenges existing knowledge or leads to the creating of new knowledge. Employing this type of message, learners integrate different points of views, extending and expanding current understanding. The purpose of this type of message was to assess the existing thoughts or belief shared by the participants and/or their culture. Questioning, argument, and reviewing are common strategies used in this type of discourse. Two examples are illustrated in Figure 6.

1 Message no. 301[Branch from no. 287]

2 Posted by Alice on Wednesday, May 21, 2003 7:26am

3 Subject Re: Writing objectives

4 Just wondering... but as we will be (and some already are) Media Specialists, we

5 will be more in the position of helping teachers make instructional objectives. We

6 will not actually write them unless asked in the in-depth level. It sounds like we are

7 support people. Do you not find that a teacher would go to their grade level teachers

8 first before they would come to the MS? 
9 Just wondering if I understand this. Oh and Karin...I'm not a teacher either...just an 10 assistant and learning all the time. Alice

11 Message no. 465

12 Posted by Jamie on Monday, May 26, 2003 11:33pm

13 Subject Is "Moderate" better?

14 Does anyone consider the fact that if ALL the teachers in a school thought that the 15 Media Specialist was a hotshot design consultant and the best thing since sliced 16 bread and decided to use this wonderful resource, that the SLMS would do 17 nothing but consult? What would happen to reading promotion, and materials 18 review and selection, and book fairs, and cataloging, and collaboration, and all the 19 other things that we hardly have time for when the teachers DON'T want us as 20 design consultants too? Maybe we don't want to reach the in-depth level, and the 21 moderate level is better?

22 Jamie

Figure 6. An Example of an Original Reformulation Message

In the first example (message 301), Alice provided her understanding of the topic (i.e., identify instructional objectives) from her context (i.e., from Media Specialist viewpoint). In this message, by providing a different perspective, Alice was trying to test the shared meaning in student participants group (i.e., role of media specialist) against her experience (lines 4-8). In the next example (message 465), Jamie framed a question to examine the concepts in practice (i.e., feasibility of moderate level analysis). Similar to the first example, this type of message generated discussion that tested the original message against personal experience. The nature of messages is alike; by assessing the original message from multiple perspectives, consequently, students may reach a general conclusion. On that account, the expected result of discussion is collective knowledge (i.e., integrated, expanded, and extended idea). Figure 7 provides an example of how collective knowledge was built during a discussion.

1 Message no. 544[Branch from no. 531]

2 Posted by Alice on Wednesday, May 28, 2003 2:51pm

3 Subject Re: Instructional Design

4 Well I guess I go with the consensus...moderate level of involvement is what I see 5 more of.

6 With all the other hats a Media Specialist wears, I can see how being an

7 instructional consultant would be a great task to achieve. I think that "no

8 involvement" should not even be on the ICAC chart...imagine not being involve in

9 the needs of the school...I can't. In the books we would review, order, and

10 catalog...should enhance the children's learning in some way or just have for the 11 love of reading. I know many Media Specialists do extensive in-service classes to 12 inform the teachers. I see myself, when I become a MS, doing the in-service 13 classes the most. I know Turner feels these are at the in-depth level, but I think 14 this is where we could reach our largest audiences. And if the teacher would like 15 extra help or information...so be it...I see time as our only restriction on what we 
16 can do and accomplish. Our time and the teacher's time to collaborated on lessons, 17 ideas, and learning strategies is limited. I did like Annette's idea of the ID in the 18 summer...though I must say I do enjoy my summers off with my kids. I would feel 19 comfortable doing any of the activities at any level (except no involvement). If I 20 am able to do initial level work with some teachers, because that is all they desire, 21 well that is as good as it will get with them.

22 Others will want (or require) the moderate or in-depth level...which is also fine. 23 We are there to help the teachers and students find the resources they need....and 24 one resource is us. Alice

Figure 7. An Example of Convergence in an Original Reformulation Message

In this example (message 544), Alice explicitly employed the word "consensus" by summarizing previous discussions (line 4) (i.e., consider moderate level as negotiated meaning from Media Specialist's angle). She substantiated her perspective with the textbook (line 13) as well as other student participant's viewpoints by mentioning Annette's message in a different thread of the discussion (lines 17-18), Alice's message also illustrates how participants cross-referred the threaded discussion.

\section{E. Re-direction}

Re-direction can be described as a type of discourse that alters or modifies goals. Within the same discussion thread, participants, including instructors and students, can modify the goals of the discussion. A redirection may facilitate and lead to new discussion as it evolves over time. Summary, evaluation, monitoring, and questioning are strategies often used in this type of discourse. This type of message tended to summarize and evaluate the previous discussion and we can expect result of discussion as emerging themes (i.e., goals), when the discussion can be continued. Figure 8 illustrates this type of discourse.

Message no. 366[Branch from no. 356]

Posted by Julie on Thursday, May 22, 2003 6:29pm

Subject Re: What about those strategies?

Alice, you hit a sore spot with me when you mentioned how the county curriculum really pushes you to fit a certain amount of information in a limited time frame.

It makes it even more difficult when your learners come to you with varying amounts of learning tools. I wish that some day public schools could concentrate more on depth of understanding instead of breadth. I think that we would have fewer students "skimming by" if we gave them opportunities to learn information in a multitude of ways. Sometimes you just need time to digest concept before the light bulb goes off. Gagne's model really helps to visualize how the steps of your lesson must build on one another to reach your goal. One of the real burdens on teachers today is to find ways to teach the required material in the allotted time without "losing" any of the learners. Julie

[discussion between these two messages by others omitted] 
1 Message no. 424[Branch from no. 366]

2 Posted by Jo on Saturday, May 24, 2003 8:15am

3 Subject Re: What about those strategies?

4 Julie -

5 Your message made me wonder how we might do what you suggested — go for 6 depth vs. breadth? Do you think that it would work if we allowed kids to start 7 focusing on particular interests earlier? Anyone have any thoughts on this? $8 \mathrm{Jo}$

Figure 8. An Example of a Re-direction Message

Jo, the university instructor, reviewed Julie's message and summarized the main point from Julie's message for further discussion. By asking a question, she opened the discussion to all participants and redirected the topics of discussion (i.e., depth vs. breadth). As we described earlier in 'description/redescription' category, in terms of planning, Jo provided guiding questions on the topic (i.e., instructional strategies) with resources in the beginning of the discussion, and with continuing discussion, she tried to achieve different goals in terms of quality (i.e., in depth examination of different meaning).

She introduced the new topics and encouraged the students to move forward to a different level of discussion. This type of message is based on a previous discussion, and is typically created at the end of a discussion. Evidence of re-direction messages was not consistently represented in the data; therefore, we cannot conclude that a discussion will always continue through the use of redirected goal(s).

\section{DISCUSSION AND IMPLICATIONS}

This study explored the nature of group interaction and discourse in a WBLE supported by computer conferencing. Five different types of online discourse were identified in this study. The types of discourse explain how learners defined the goals for the discussion, represented individual understanding, connected to collaborative efforts, tested shared meaning, and redirected the discussion. As described in the data presentation, dynamic interactions amongst participants can only be described and understood in association with the individual message in relation to others. Therefore, each message type is explained in relationship to the context of the discussion.

As we described earlier, it is meaningful to consider different dimensions in examining discourse generated via asynchronous discussion. Understanding the technological dimension helped to guide us in deciding the unit of analysis for this study. The technological affordances of the system used can influence flows of interaction, such as turn taking $([27,34,35])$. Thus, we investigated the threads of the discussion to observe how a message(s) contributed to the ongoing discussion. Closely related to social dimension, we believe that a simple analysis of individual message does not support an examination of how meaning was situated in a social process of learning. Collaborative efforts made by participants to be engaged in the discussion were critical for developing understanding of the process. We discuss each type of online discourse from situated learning perspective in the following paragraphs.

In this study, the goal setting message contributed to initiating and continuing the discussion, leading to shared goals. Without shared goals, a group of learners may not be able to proceed in their discussion [30, 45]. In this specific context, the pre-planned goals of the instructors were represented by the instructors as well as learner participants. The goal was shared in the ongoing discussion. The formal education context 
Building Understanding in Asynchronous Discussions: Examining Types of Online Discourse

also contributed to participant contributions. One of the requirements for the course was participation in the online discussions. Engagement of the learners in the learning process was presumed to be a given, and, participation in the discussion was the responsibility of each learner. It is important to note, however, that participation varied amongst learners with some engaging more actively in the process than others.

The reflection message allowed participants to represent individual understanding. Understanding is a matter of connecting information [20]. By representing individual understanding in a social context, participants were able to become members of a learning community that might be very different from the learning community with which they are most familiar [30]. This type of message was often used to provide an answer to a guiding question. When a reflection message is posted, it is focused on how the individual understands the problem. However, the reflection messages generated by participants are shared. Consequently, participants have access to multiple perspectives on how others think. This may enable others to extend or even change their perceptions [44, 46].

The connection message was observed when an individual reflection was situated within the collaborative context. Elaboration on each other's perspective creates links between individual ideas [47], allowing learners to demonstrate their ability to see the essence of the problem (as reflected in the discussion topic) based on deep understanding of the context [48]. It is the responsibility of the individual to situate the meanings in the social context by integrating new ideas with previous knowledge and experience. Yet, the "control" of the validation of meaning (i.e., mutual agreement) exists in the group, not the individuals [48]. Therefore, in the asynchronous discussion, individual accountability and participants' interdependent relationships coexist.

The original reformulation message challenged existing knowledge during the discussion explored in this study. In an original reformulation message, participants integrated various perspectives, mutually extending and expanding current understanding. Collective knowledge is not always identified in one thread. However, as discussed earlier, it is possible to observe how an individual participant's message indicated the convergence (i.e., collective knowledge). According to the literature, the crux of collaborative learning is the ongoing process of inquiry, not that participants inevitably create new knowledge [49]. One indicator of the ongoing process of inquiry is that participants perceive that some advance has been made in existing knowledge and that they believe they learned through the discussion [49]. This was demonstrated in this study.

The re-direction message indicated qualitative change in the discussion in this study. Re-direction messages were often found at the end of the discussion. This type of message contributed to the summarization and evaluation of the previous discussion. The re-direction messages were often identified in the data; however, the discussion was not always continued. As described, this type of message commonly occurred at the end of the discussion. Consequently, with given time framework of the formal education context, the discussion may not proceed and the students could already have moved to next discussion section [27].

\section{METHODOLOGICAL CONSIDERATIONS}

Physical access to data for interaction analysis is relatively easy. Transcripts of online discussions are readily accessible since the written data is complied as text during and after the discussion. However, given the rich and complex nature of the data, conceptual access may not be so easy. One single method of examining the data does not appear to be sufficient; rather multiple methods appear to be useful in gaining a richer understanding of the interactions that occur in online discussions. 
Analyzing the transcripts of the asynchronous discussion board was truly a challenging task, perhaps the most challenging task in this study. The text analyzed was rich and complex, yet the analysis was limited in some ways. By examining the text as generated by the system, we were able to investigate how particular ideas and/or uses of strategies might be employed and transformed by discussion participants over time. However, this strategy is not sufficient for capturing the evolution of ideas and building of understanding by participants [50]. If we want to explore the creation of knowledge through online discussions, there are many questions that need to be answered, including: what is the unit of analysis (i.e., individual messages, discussion threads)? What is the best configuration of the data for exploring knowledge creation? What other types of data might be needed (by one discussion archives) to explore knowledge creation? Further research is needed to help address these and related questions.

Another challenge comes from the nature of data. Due to the asynchronicity, it is not simple to analyze the activities that occurred during the reading, writing, and posting messages outside as well as within the discussion board. If we examine the evolution of a discussion over time, how might we conceptualize 'distributed' time-asynchronous participation—by the growth of discussion threads or particular themes? Or do we consider its evolution over time across participants? Future research is needed that will enable extensive data collection methods to substantiate the findings (e.g., in-depth recall interview, participant's self-reflection during and after the session, post-group discussion).

\section{IMPLICATIONS FOR FUTURE RESEARCH}

One area in need of further examination is the use of different strategies during a discussion. While multiple strategies were identified from the data, how each strategy contributed to the discussion was not fully explored. Future research needs to focus on how various strategies are employed in multiple contexts and how they might contribute to the discussion. For example, question [51] and argument [52] type messages are well described in recent research. A more detailed analysis of discourse strategies may extend our understanding of how discussion supports the learning process and what supports can be created in an asynchronous discussion. It would also extend the ideas how each strategy functioned in a different type of discourse.

Further research is also needed to connect existing social learning theories to each type of message identified from the data. The purpose of this study was not to prescribe the types of messages found in all asynchronous discussions. Rather, we strived to describe the types of discourse created while participants were engaged in and continued the discussion. However, we also believe that collaborative learning theories (i.e., constructivism) and situated cognition perspective support to explain how learning occurs in this specific context. Tied to specific theories, each type can be articulated and elaborated upon to describe the learning process. Further research is needed to extend this process.

\section{IMPLICATIONS FOR PRACTICE}

One area in need of further investigation related to the co-construction of knowledge is the consideration of the impact of who is implementing the online course. It could be argued that through collaborative tasks, the creation of shared products, and the very nature of online discussion, many online experiences are actually implemented by the participants. The official facilitator (i.e., instructor) may or may not lead or guide the discussions. Instead, the interactions may be primarily guided and facilitated by the participants. This creates a change in the pedagogical process. Therefore, we need to carefully examine existing learning theories for "best fit" in online contexts while also exploring strategies for facilitating the processes in online learning so that new theories and practices can be established [53]. 
Building Understanding in Asynchronous Discussions: Examining Types of Online Discourse

Another consideration for practice is assessment. One way to evaluate whether collaboration has occurred in a group is to assess the amount and nature of the interaction among participants. Interactions may occur when group members refer explicitly or implicitly to prior messages in a discussion, while staying on the topic. However, as indicated, the interaction does not always appear as a set of interdependent statements, direct/indirect comments for previous messages, or answers to questions. Several strategies could be employed to enable students to do self evaluation as individuals, self-evaluation as a group, and a comparison between students' performance and the class objectives. How the students share the responsibility of collaborative work and peer evaluation on contribution to the discussion also needs to be addressed in the process of assessment [54]. In that way, we may be able to evaluate participation as a social process, which should not necessarily be measured by the total number and length of all the messages sent and received by all members of the group.

Another suggestion for practice is training participants in how to communicate online. As the literature indicated, not all instructors and students are well prepared for implementation of collaborative learning $[55,56]$. Fostering learners' capability to engage in online discussion may contribute to successful learning experience [57]. The strategies for fostering learners' readiness for online collaborative learning should include such things as developing collaborative skills [54] as well as technology competency [56]. The facilitators are also responsible for the preparation of necessary online learning skills related to tools, materials, and resources.

\section{CONCLUSION}

As the technology continues to evolve, and we become more skilled with communicating online, the exploration of a variety of methods for studying these environments will continue to increase. Further, we will likely need new teaching and learning strategies to fully engage the capabilities of the systems, both the human and technology. Continuing our exploration is vital if we are to realize the full potential of these learning contexts.

\section{REFERENCES}

1. Bruckman, A. The future of e-learning communities. Communication of the ACM 45(4): 60-63, 2002.

2. de Jong, F., E. Veldhuis-Diermanse and G. Lutgens. Computer-supported collaborative learning in university and vocational education, In: Koschmann, T., R. Hall, and N. Miyake (Eds.), CSCL2: Carrying forward the conversation, 111-128. Mahwah, NJ: Lawrence Erlbaum, 2002.

3. Kemery, E. R. Developing on-line collaboration, In: A. Aggarwal (Ed.), Web-based learning and teaching technologies: Opportunities and challenges, 227-245. Hershey, PA: Idea Group, 2000.

4. Rogers, J. Communities of practice: A framework for fostering coherence in virtual learning communities. Educational Technology \& Society 3(3): 2000. http://grouper.ieee.org/groups/ifets /periodical/vol 3 2000/e01.html.

5. Islas, J. R. L. Collaborative learning at Monterrey Tech-Virtual University. In: T. Duffy and J. R. Kirkley (Eds.), Learner-centered theory and practice in distance education: Cases from higher education, 297-319. Mahwah, NJ: Lawrence Erlbaum, 2004.

6. Kanuka, H. and T. Anderson. Online social interchange, discord, and knowledge construction. Journal of Distance Education 13(1): 1998. http://cade.athabascau.ca/vol13.1/kanuka.html.

7. Järvelä, S. and P. Häkkinen. Web-based cases in teaching and learning - The quality of discussions and a stage of perspective taking in asynchronous communication. Interactive Learning Environments 10(1): 1-22, 2002. 
8. Anderson, T. Modes of interaction in distance education: Recent developments and research questions, In: M. G. Moore, and W. G. Anderson (Eds.), Handbook of distance education, 129-144. Mahwah, NJ: Lawrence Erlbaum, 2003.

9. Hill, J. R., D. Wiley, L. M. Nelson and S. Han. Exploring research on Internet-based learning: From infrastructure to interactions, In: D. H. Jonassen (Ed.), Handbook of Research on Educational Communications and Technology, $2^{\text {nd }}$ ed., 433-460. Mahwah, NJ: Lawrence Erlbaum, 2004.

10. Marra, R. M., J. L. Moore and A. K. Kimczak. Content analysis of online discussion forums: A comparative analysis protocols. Educational Technology Research \&Development 52(2): 23-40, 2004.

11. Alavi, M. and D. Duftner. Technology-mediated collaborative learning: A research perspective, In: S. R. Hiltz and R. Goldman (Eds.), Learning together online: Research on asynchronous learning networks, 191-213. Mahwah, NJ: Lawrence Erlbaum, 2005.

12. Järvelä, S. and $\mathbf{P}$. Häkkinen. The levels of Web-based discussions: Using perspective-taking theory as an analytical tool. In: H. van Oostendorp (Ed.), Cognition in a digital world. Mahwah, NJ: Lawrence Erlbaum, 2003.

13. Brown, J. S., A. Collins and S. Duguid. Situated cognition and the culture of learning, Educational Researcher 18(1): 32-42, 1989.

14. Wenger, E. Communities of practices: Learning, meaning, and identity. New York: Cambridge University Press, 1998.

15. Salomon, G. No distribution without individuals' cognition: A dynamic interactional view, In: G. Salomon, (Ed.), Distributed cognition: Psychological and educational consideration, 111-138. New York: Cambridge University Press, 1993.

16. Pea, R. D. Practices of distributed intelligence and designs for education, In: G. Salomon (Ed.), Distributed cognitions: Psychological and educational considerations, 47-87. New York: Cambridge University Press, 1993.

17. Pea, R. D. The social and technological dimensions of scaffolding and related theoretical concepts for learning, education, and human activity. The Journal of the Learning Sciences 13(3): 423-451, 2004.

18. Barab, S. A. and P. A. Kirschner. Guest editor's introduction: Rethinking methodology in the learning sciences. The Journal of the Learning Sciences 10 (1 \& 2): 5-15, 2001.

19. Kirshner, D. and J. A. Whitson. Editors' introduction to situated cognition, In: D. Kirshner and J. A. Whitson (Eds.), Situated cognition: Social, semiotic, and psychological perspectives, 1-16. Mahwah, NJ: Lawrence Erlbaum, 1997.

20. Salomon, G. Technology's promises and dangers in a psychological and educational context. Theory into Practice 37(1): 3-10, 1998.

21. Chee-Kit, L. Communication techniques, In: H. H. Aderlsberger, B. Collis, and J. M. Pawlowski (Eds.), Handbook of information technologies for education and training, 45-56. Berlin: SpringerVerlag, 2002.

22. Inglis, A., Ling, P. and Joosten, V. Delivering digitally: Managing the transition to the knowledge media. London: Kogan Page, 1999.

23. Ryan, S., B. Scott, H. Freeman and D. Pate. The virtual university. London: Kogan Page, 2000.

24. Zumbach, J. and P. Reimann. Combining computer supported collaborative argument and problembased learning: An approach for designing online learning environments, Paper presented at the Computer Supported Collaborative Argumentation Workshop at the CSCL 99 Conference, Stanford, California, December 1999. http://d3e.open.ac.uk/cscl99/Zumbach/Zumbach-paper.html.

25. Klemm, W. R. and J. R. Snell. Enriching computer-mediated group learning by coupling constructivism with collaborative learning. Journal of Instructional Science and Technology 1(2): 1996. http://www.usq.edu.au/electpub/e-jist/docs/old/vol1no2/article1.htm.

26. Condon, S. L. and C. G. Čech. Functional comparisons of face-to-face and computer-mediated decision making interactions, In: S. C. Herring (Ed.), Computer-mediated communication: Linguistic, social and cross-cultural perspectives, 65-80. Philadelphia, PA: John Benjamins, 1996. 
27. Davis, B. H. and J. P. Brewer. Electronic discourse: Linguistic individuals in virtual space. Albany, NY: SUNY Press, 1997.

28. Gee, J. P. Thinking, learning, and reading: The situated sociocultural mind,. In: D. Kirshner and J. A. Whitson (Eds.), Situated cognition: Social, semiotic, and psychological perspectives, 235-280. Mahwah, NJ: Lawrence Erlbaum, 1997.

29. Gee, J. P. An introduction to discourse analysis: Theory and method, London: Routledge, 1999.

30. Bruffee, K. A. Collaborative learning: Higher education, interdependence, and the authority of knowledge, $2^{\text {nd }}$ ed. Baltimore, MD: The Johns Hopkins University, 1999.

31. Gill, R. Discourse analysis, In: M. W. Bauer and G. Gaskell (Eds.), Qualitative researching with text, image and sound: A practical handbook, 172-190. Thousand Oaks, CA: Sage, 2000.

32. Mann, C. and F. Stewart. Internet communication and qualitative research: A handbook for researching online. Thousand Oaks, CA: Sage, 2000.

33. Yates, S. Oral and written linguistic aspects of computer conferencing: A corpus based study, In: S. C. Herring (Ed.), Computer-mediated communication: Linguistic, social and cross-cultural perspectives, 29-46. Philadelphia, PA: John Benjamins, 1996.

34. Garcia, A. C. and J. B. Jacobs. The eyes of the beholder: Understanding the turn-taking system in quasi-synchronous computer-mediated communication. Research on language and social interaction 32(4): 337-368, 1999.

35. Hutchby, I. Conversation and technology: From the telephone to the Internet. Cambridge, UK: Polity, 2001.

36. Goetz, J. P. and M. D. LeCompte. Ethnography and qualitative design in educational research. New York: Academic Press, 1984.

37. Patton, M. Q. Qualitative research and evaluation methods, $3^{\text {rd }}$ ed. Thousand Oaks, CA: Sage, 2002.

38. Resnick, L. M., Pontecorvo, C. and Säljö, R. Discourse, tools, and reasoning: Essays on situated cognition, In: L. B. Resnick, R. Säljö, C. Pontecorvo, and B. Burge (Eds.), Discourse, tools, and reasoning: Essays on situated cognition, 1-20. Berlin: Springer-Verlag, 1997.

39. Gunawardena, C. N., C. A. Lowe and T. Anderson. Analysis of global online debate the development of an interaction analysis model for examining social construction of knowledge in computer conferencing. Journal of Educational Computing Research 17(4): 397-431, 1997.

40. MacLure, M. Discourse in educational and social research. Philadelphia, PA: Open University Press, 2003.

41. Wood, L. A. and R. O. Kroger. Doing discourse analysis: Methods for studying action in talk and text. Thousand Oaks, CA: Sage, 2000.

42. Cecez-Kecmanovic, D. and C. Webb. A critical inquiry into web-mediated collaborative learning. In A. Aggarwal (Ed.), Web-based learning and teaching technologies: Opportunities and challenges, 307-326. Hershey, PA: Idea Group Publishing, 2000.

43. Bereiter, C. and M. Scardamalia. The psychology of written composition. Hillsdale, NJ: Lawrence Erlbaum, 1987.

44. Levin, B. B. Analysis of the content and purpose of four different kinds of electronic communications among preservice teachers. Journal of Research on Computing in Education 32(1): 139-156, 1999.

45. Palloff, R. M. and Pratt, K. Building learning communities in cyberspace: Effective strategies for the online classroom. San Francisco, CA: Jossey-Bass, 1999.

46. Bell, P. Using argument map presentations to make thinking visible for individuals and groups, In: T. Koschmann, R. Hall, and N. Miyake (Eds.), CSCL2: Carrying forward the conversation, 449-485. Hillsdale, NJ: Lawrence Erlbaum, 2002.

47. Harasim, L. What makes online learning communities successful?: The role of collaborative learning in social and intellectual development, In: C. Vrasidas and G. V. Glass (Eds.), Distance education and distributed learning, 181-200. Greenwich, CO: Information Age, 2002.

48. Garrison, D. R. Critical thinking and self-directed learning in adult education: An analysis of responsibility and control issues. Adult Education Quarterly 42(3): 136-148, 1992. 
49. Scardamalia, M. and C. Bereiter. Technologies for knowledge-building discourse. Communications of the ACM 37(5): 37-41, 1993.

50. Sutton, L. A. The principles of vicarious interaction in computer-mediated communications. International Journal of Educational Telecommunication 7(3): 223-242, 2001.

51. Ge, X. and S. M. Land. A conceptual framework for scaffolding ill-structured problem-solving processes using question prompts and peer interaction. Educational Technology Research \& Development 52(2): 5-20, 2004.

52. Marttunen, M. and L. Laurinen. Quality of students' argumentation by e-mail. Learning Environments Research 5: 99-123, 2001.

53. Miller, S. M. and K. L. Miller. Theoretical and practical considerations in the design of Web-based instruction. In: B. Abbey (Ed.), Instructional and cognitive impacts of Web-based education, 156177. Hershey, PA: Idea Group Publishing, 2000.

54. Johnson, D. W. and Johnson, R. Assessing students in groups: Promoting group responsibility and individual accountability. Thousand Oaks, CA: Corwin Press, 2004.

55. Brandon, D. P. and A. B. Hollingshead. Collaborative learning and computer-supported groups. Communication Education 48(2): 109-129, 1999.

56. Kemery, E. R. Developing on-line collaboration, In: A. Aggarwal. (Ed.), Web-based learning and teaching technologies: Opportunities and challenges, 227-245. Hershey, PA: Idea Group Publishing, 2000.

57. Marjanovic, O. Learning and teaching in a synchronous collaborative learning environment. Journal of Computer Assisted Learning 15(2): 129-138, 1999.

\section{ABOUT THE AUTHORS}

Seungyeon Han, Ph.D., is a Professor of Educational Technology at Hanyang Cyber University, Seoul, Korea. She received her Ph.D. in Instructional Technology from the University of Georgia (2005). Her current research focuses on Web-based collaborative learning, community building in virtual environments, and qualitative inquiry in technology-mediated discourse.

Janette R. Hill is an associate professor, University of Georgia, College of Education, Department of Educational Psychology and Instructional Technology. Prior to coming to the University of Georgia, Dr. Hill held academic appointments at the University of Northern Colorado and Georgia State University. Dr. Hill's research interests include community building, resource-based learning and Web-based learning environments. Dr. Hill holds a master's in library and information science and a doctoral degree in Instructional Systems Design, both from Florida State University. Her articles have appeared in research and professional publications including Educational Technology Research and Development and The Internet and Higher Education. 